\title{
SYMMETRY FOR A DIRICHLET-NEUMANN PROBLEM ARISING IN WATER WAVES
}

\author{
RAFAel de la Llave ANd EnRico Valdinoci
}

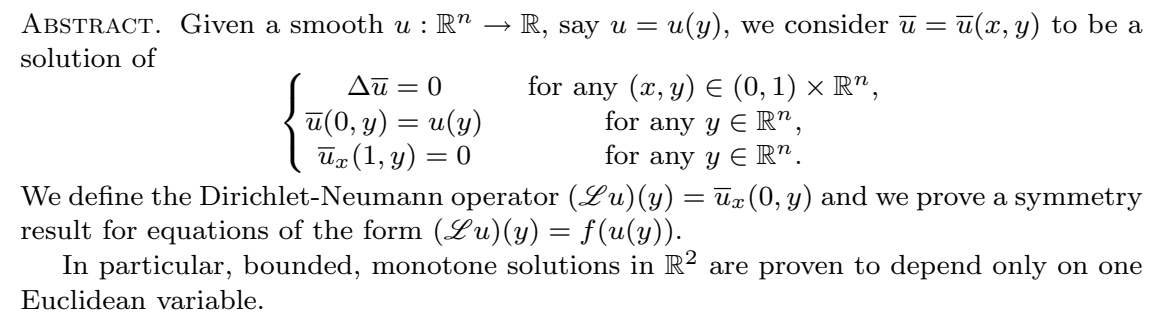

\section{Introduction}

The aim of this paper is to provide a symmetry result for a Dirichlet-Neumann problem.

Our set up is the following. We consider the slab $[0,1] \times \mathbb{R}^{n}$, endowed with coordinates $x \in[0,1]$ and $y \in \mathbb{R}^{n}$.

We define the operator $\mathscr{L}$ as follows. Given a smooth $u$, which will be taken to be bounded together with its derivatives, we define $\bar{u}(x, y) \in C^{2}\left((0,1) \times \mathbb{R}^{n}\right) \cap C^{1}([0,1] \times$ $\mathbb{R}^{n}$ ) to be the solution of

$$
\left\{\begin{array}{c}
\Delta \bar{u}=0 \quad \text { in }(0,1) \times \mathbb{R}^{n}, \\
\bar{u}(0, y)=u(y), \\
\bar{u}_{x}(1, y)=0 .
\end{array}\right.
$$

As customary, the subscript denotes the partial derivative and $\Delta \bar{u}=\bar{u}_{x x}+\bar{u}_{y_{1} y_{1}}+$ $\cdots+\bar{u}_{y_{n} y_{n}}$ is the Laplace operator. The problem in (1) is well-posed and it possesses nice regularity properties, due to the elliptic PDE theory (see, e.g., Theorems 6.6 and 6.26 in [23]). Then, we define

$$
(\mathscr{L} u)(y)=\bar{u}_{x}(0, y) .
$$

The linear operator $\mathscr{L}$ may also be written in the harmonic analysis setting. That is, if $\mathscr{F}$ denotes the Fourier transform in the $y$ variables (and the transformed frequency variables are called $\xi \in \mathbb{R}^{n}$ ), we have that

$$
\mathscr{L} u=\mathscr{F}^{-1}\left(|\xi| \frac{e^{-|\xi|}-e^{|\xi|}}{e^{-|\xi|}+e^{|\xi|}}(\mathscr{F} u)(\xi)\right),
$$

up to a normalization factor.

Received by the editors October 21, 2008. 
From (2), we may say that the symbol of the operator $\mathscr{L}$ in Fourier space is

$$
|\xi| \frac{e^{-|\xi|}-e^{|\xi|}}{e^{-|\xi|}+e^{|\xi|}}
$$

Though Fourier analysis will not explicitly play much of a role in this paper, it is convenient to keep in mind that, for large frequencies $\xi$, (3) is asymptotic to the symbol of the square root of the Laplacian.

The operator $\mathscr{L}$ arises in the theory of water waves of irrotational, incompressible, inviscid fluids in the small amplitude, long wave regime $[35,38,37,12,9,28,13,15$, 11, 10, 22, 25, 29].

Related nonlocal operators are studied in flame propagation and semipermeable membranes [3], in optimization [17], in relation with the ultrarelativistic limit of quantum mechanics [20], in the theory of quasi-geostrophic flows [27, 7] in inverse spectral and multiple scattering problems $[16,6,24]$ and in the thin obstacle problem [4].

Of course, these operators are also a classical topic in harmonic analysis and in singular integral theory $[26,32]$.

The main result that we prove is the following:

Theorem 1. Let $f \in C^{1}(\mathbb{R})$.

Let $u$ be a bounded solution of $(\mathscr{L} u)(y)=f(u(y))$ for any $y \in \mathbb{R}^{n}$.

Suppose that

$$
u_{y_{n}}(y)>0 \text { for any } y \in \mathbb{R}^{n}
$$

and that there exists $C>0$ such that

$$
\int_{x \in[0,1]} \int_{|y| \leq \tau}\left|\nabla_{y} \bar{u}(x, y)\right|^{2} d y d x \leq C \tau^{2}
$$

for any $\tau \geq C$.

Then, there exist $u_{o}: \mathbb{R} \rightarrow \mathbb{R}$ and $\omega \in \mathrm{S}^{n-1}$ such that

$$
u(y)=u_{o}(\omega \cdot y) \quad \text { for any } y \in \mathbb{R}^{n} .
$$

We remark that (6) states that $u$ depends only on one Euclidean variable up to rotation (equivalently, $u$ is constant in the directions orthogonal to $\omega$ ). In this sense, Theorem 1 is inspired by a celebrated conjecture for monotone, entire solutions of elliptic PDEs in [14].

In particular, as a consequence of Theorem 1, we obtain the following result for $n=2$ :

Corollary 2. Let $f \in C^{1}(\mathbb{R})$.

Let $u$ be a bounded solution of $(\mathscr{L} u)(y)=f(u(y))$ for any $y \in \mathbb{R}^{2}$, such that

$$
u_{y_{2}}(y)>0 \text { for any } y \in \mathbb{R}^{2} \text {. }
$$

Then, there exist $u_{o}: \mathbb{R} \rightarrow \mathbb{R}$ and $\omega \in \mathrm{S}^{1}$ such that

$$
u(y)=u_{o}(\omega \cdot y) \quad \text { for any } y \in \mathbb{R}^{2} .
$$


The analogy between the result in Corollary 2 and the conjecture for entire, monotone, bounded solutions of semilinear elliptic PDEs in [14] is manifest. We would like to mention that [8] presents rigidity results for nonnegative, localized solitary waves and [36] contains symmetry results for different fluid dynamics problems also inspired by $[14]$.

The proofs of the above results are suitable modifications of the work done in [31] and they are based on a geometric inequality (namely (25) below) which may be seen as an extension of a similar one obtained, in a different setting, by [33, 34].

The idea of using geometric inequalities to derive symmetry results was also used in $[18,19]$.

We would also like to recall that the first symmetry result for boundary reaction PDEs was obtained, with different methods, in [2] for the halfspace (such setting as a fractional operator, corresponds to the square root of the Laplacian). For related results, see also $[30,5]$.

Below are the details of the proofs of Theorem 1 and Corollary 2.

\section{Proofs of the main results}

In order to prove Theorem 1, we need some preliminary observations:

Lemma 3 (Weak form of the equation). Let $\bar{u}$ be a solution of (1).

Then, for any $\phi \in C_{0}^{\infty}\left(\mathbb{R}^{n+1}\right)$,

$$
-\int_{\{0\} \times \mathbb{R}^{n}} \phi(\mathscr{L} u)=\int_{[0,1] \times \mathbb{R}^{n}} \nabla \phi \cdot \nabla \bar{u} .
$$

Proof. Given $\phi \in C_{0}^{\infty}\left(\mathbb{R}^{n+1}\right)$, we denote by $\mathscr{D}_{\phi}$ the intersection between a ball containing the support of $\phi$ and $[0,1] \times \mathbb{R}^{n}$. We also denote by $\nu$ the exterior normal of $\partial \mathscr{D}_{\phi}$, which is well-defined almost everywhere.

Then, we have

$$
\begin{aligned}
0=\int_{[0,1] \times \mathbb{R}^{n}} \Delta \bar{u} \phi=\int_{\mathscr{D}_{\phi}}(\operatorname{div}(\phi \nabla \bar{u})-\nabla \phi \cdot \nabla \bar{u}) \\
=\int_{\partial \mathscr{D}_{\phi}} \phi \nabla \bar{u} \cdot \nu-\int_{\mathscr{D}_{\phi}} \nabla \phi \cdot \nabla \bar{u} \\
=-\int_{\{0\} \times \mathbb{R}^{n}} \phi(\mathscr{L} u)-\int_{[0,1] \times \mathbb{R}^{n}} \nabla \phi \cdot \nabla \bar{u} .
\end{aligned}
$$

Lemma 4 (Weak form of the linearized equation). Let $f \in C^{1}(\mathbb{R})$ and let $u$ be $a$ solution of $(\mathscr{L} u)(y)=f(u(y))$ for any $y \in \mathbb{R}^{n}$.

Assume that $u(y)=\bar{u}(0, y)$, with $\bar{u}$ as in (1).

Given $i=1, \ldots, n$, we have that

$$
-\int_{\{0\} \times \mathbb{R}^{n}} \psi f^{\prime}(u) u_{y_{i}}=\int_{[0,1] \times \mathbb{R}^{n}} \nabla \psi \cdot \nabla \bar{u}_{y_{i}}
$$

for any $\psi \in C_{0}^{\infty}\left(\mathbb{R}^{n+1}\right)$. 
Proof. We start with an elementary observation about the integration by parts formula: if $\Psi \in C_{0}^{\infty}\left(\mathbb{R}^{n+1}\right)$ and $\zeta \in C^{1}\left((0,1) \times \mathbb{R}^{n}\right)$, then, for any fixed $x \in(0,1)$, the map $y \mapsto \Psi(x, y)$ belongs to $C_{0}^{\infty}\left(\mathbb{R}^{n}\right)$ and therefore

$$
-\int_{\mathbb{R}^{n}} \Psi(x, y) \zeta_{y_{i}}(x, y) d y=\int_{\mathbb{R}^{n}} \Psi_{y_{i}}(x, y) \zeta(x, y) d y .
$$

Therefore, integrating in $x$, we obtain

$$
-\int_{[0,1] \times \mathbb{R}^{n}} \Psi \zeta_{y_{i}}=\int_{[0,1] \times \mathbb{R}^{n}} \Psi_{y_{i}} \zeta .
$$

Now, we take $\psi \in C_{0}^{\infty}\left(\mathbb{R}^{n+1}\right)$ and $\phi=\psi_{y_{i}}$ in (7), we use (9) and we conclude that

$$
\begin{gathered}
-\int_{\{0\} \times \mathbb{R}^{n}} \psi f^{\prime}(u) u_{y_{i}}=-\int_{\{0\} \times \mathbb{R}^{n}} \psi(f(u))_{y_{i}}=\int_{\{0\} \times \mathbb{R}^{n}} \psi_{y_{i}} f(u) \\
=-\int_{[0,1] \times \mathbb{R}^{n}} \nabla \psi_{y_{i}} \cdot \nabla \bar{u}=\int_{[0,1] \times \mathbb{R}^{n}} \nabla \psi \cdot \nabla \bar{u}_{y_{i} .}
\end{gathered}
$$

Lemma 5 (Sign property). Let $v \in C^{2}\left((0,1) \times \mathbb{R}^{n}\right) \cap C^{1}\left([0,1] \times \mathbb{R}^{n}\right)$, with finite $\|v(0, \cdot)\|_{C^{2, \alpha}\left(\mathbb{R}^{n}\right)}$, satisfy

$$
\left\{\begin{array}{c}
\Delta v=0 \\
v_{x}(1, y)=0 .
\end{array} \text { in }(0,1) \times \mathbb{R}^{n},\right.
$$

If $v(0, y)>0$ for any $y \in \mathbb{R}^{n}$, then $v(x, y)>0$ for any $x \in[0,1)$ and any $y \in \mathbb{R}^{n}$.

Proof. By the strong maximum principle, it is enough to show that $v \geq 0$ in $(0,1) \times \mathbb{R}^{n}$.

Thus, we argue by contradiction and we suppose that $v(\bar{x}, \bar{y})<0$ for some $(\bar{x}, \bar{y}) \in$ $(0,1) \times \mathbb{R}^{n}$.

Hence, by the maximum principle,

$$
\inf _{(x, y) \in(0,1) \times \mathbb{R}^{n}} v(x, y)=\inf _{y \in \mathbb{R}^{n}} v(1, y)<0 .
$$

Therefore, we take a sequence $y_{j}$ such that

$$
\lim _{j \rightarrow+\infty} v\left(1, y_{j}\right)=\inf _{y \in \mathbb{R}^{n}} v(1, y)<0 .
$$

We define

$$
v_{j}(x, y)=v\left(x, y_{j}+y\right) .
$$

By elliptic regularity [23], up to an even reflection across $\{x=1\}$, we have that $\|v\|_{C^{2, \beta}\left((0,1) \times \mathbb{R}^{n}\right)}$ is bounded, for some $\beta \in(0,1)$. So, up to subsequences $v_{j}$ converges locally uniformly to some $w$, together with its first two derivatives.

Thus, (10) gives that

$$
\left\{\begin{array}{c}
\Delta w=0 \quad \text { in }(0,1) \times \mathbb{R}^{n}, \\
w_{x}(1, y)=0 .
\end{array}\right.
$$

Also

$$
w(0, y)=\lim _{j \rightarrow+\infty} v\left(0, y_{j}+y\right) \geq 0
$$

and

$$
w(1,0)=\lim _{j \rightarrow+\infty} v\left(1, y_{j}\right)=\inf _{y \in \mathbb{R}^{n}} v(1, y) .
$$


From (13), we have that

$$
w(1,0)<0
$$

and that

$$
w(1,0) \leq v\left(1, y+y_{j}\right)=v_{j}(1, y) \text { for any } y .
$$

Accordingly, (15) gives that

$$
w(1,0) \leq w(1, y) \text { for any } y .
$$

Then, making use of (11), (12), (14), (16) and the maximum principle, we have that

$$
\inf _{(x, y) \in(0,1) \times \mathbb{R}^{n}} w(x, y)=\inf _{y \in \mathbb{R}^{n}} w(1, y)=w(1,0) .
$$

Consequently, Hopf principle and (11) imply that $w$ is constant.

This constant must be nonnegative, due to (12), but this is in contradiction with (14).

Corollary 6 (Monotonicity property I). Let $\bar{u}$ be a solution of (1).

$$
\text { If } \bar{u}_{y_{n}}(0, y)>0 \text { for any } y \in \mathbb{R}^{n} \text {, then } \bar{u}_{y_{n}}(x, y)>0 \text { for any }(x, y) \in[0,1) \times \mathbb{R}^{n} \text {. }
$$

Proof. Set $v=\bar{u}_{y_{n}}$ and employ Lemma 5 .

Lemma 7 (Monotonicity property II). Let $\bar{u}$ be a solution of (1). If $\bar{u}_{y_{n}}(x, y)>0$ for any $(x, y) \in[0,1) \times \mathbb{R}$, then

$$
\int_{[0,1] \times \mathbb{R}^{n}}|\nabla \varphi|^{2}+\int_{\{0\} \times \mathbb{R}^{n}} f^{\prime}(u) \varphi^{2} \geq 0
$$

for any $\varphi \in C_{0}^{\infty}\left(\mathbb{R}^{n+1}\right)$.

Proof. The following is a variation of a classical argument (see [1]). Possibly after approximation, we may take $i=n$ and $\psi=\varphi^{2} / \bar{u}_{y_{n}}$ in (8). Thus, making use of the Cauchy-Schwarz inequality we obtain

$$
-\int_{\{0\} \times \mathbb{R}^{n}} f^{\prime}(u) \varphi^{2}=\int_{[0,1] \times \mathbb{R}^{n}}\left(\frac{2 \varphi \nabla \varphi \cdot \nabla \bar{u}_{y_{n}}}{\bar{u}_{y_{n}}}-\frac{\varphi^{2}\left|\nabla \bar{u}_{y_{n}}\right|^{2}}{\bar{u}_{y_{n}}^{2}}\right) \leq \int_{[0,1] \times \mathbb{R}^{n}}|\nabla \varphi|^{2} .
$$

With the above observations, we can now complete the

Proof of Theorem 1. We take $\bar{u}$ as in (1), such that $u(y)=\bar{u}(0, y)$. We also write $X=(x, y) \in[0,1] \times \mathbb{R}^{n}$. Notice that, in this notation

$$
\nabla=\left(\partial_{x}, \partial_{y_{1}}, \ldots, \partial_{y_{n}}\right)=\left(\partial_{X_{1}}, \ldots, \partial_{X_{n+1}}\right) .
$$

Given $\eta \in C_{0}^{\infty}\left(\mathbb{R}^{n}\right)$, we choose $\psi=\bar{u}_{y_{i}} \eta^{2}$ in (8). By summing over the index $i$, and using the notation in (18), we obtain, after a simple calculation,

$$
(19)-\int_{\{0\} \times \mathbb{R}^{n}} f^{\prime}(u)\left|\nabla_{y} u\right|^{2} \eta^{2}=\int_{[0,1] \times \mathbb{R}^{n}}\left(\eta^{2} \sum_{\substack{2 \leq i \leq n+1 \\ 1 \leq j \leq n+1}}\left(\partial_{X_{i} X_{j}} \bar{u}\right)^{2}+\frac{1}{2} \nabla \eta^{2} \cdot \nabla\left|\nabla_{y} \bar{u}\right|^{2}\right) .
$$

Furthermore, by (4) and Corollary 6 , we have that $\bar{u}_{y_{n}}(x, y)>0$ for any $(x, y) \in$ $[0,1) \times \mathbb{R}^{n}$. 
This and Lemma 7 imply that (17) holds true. Accordingly, given $\eta \in C_{0}^{\infty}\left(\mathbb{R}^{n+1}\right)$, possibly after an approximation argument, we may take $\varphi=\left|\nabla_{y} \bar{u}\right| \eta$ in (17) and conclude that

$\int_{[0,1] \times \mathbb{R}^{n}}\left(|\nabla \eta|^{2}\left|\nabla_{y} \bar{u}\right|^{2}+\left.\eta^{2}|\nabla| \nabla_{y} \bar{u}\right|^{2}+\frac{1}{2} \nabla \eta^{2} \cdot \nabla\left|\nabla_{y} \bar{u}\right|^{2}\right) \geq-\int_{\{0\} \times \mathbb{R}^{n}} f^{\prime}(u)\left|\nabla_{y} \bar{u}\right|^{2} \eta^{2}$.

As a consequence of this and of (19), some interesting cancellations give that

$$
\int_{[0,1] \times \mathbb{R}^{n}} \eta^{2}\left(\sum_{\substack{2 \leq i \leq n+1 \\ 1 \leq j \leq n+1}}\left(\partial_{X_{i} X_{j}} \bar{u}\right)^{2}-|\nabla| \nabla_{y} \bar{u}||^{2}\right) \leq \int_{[0,1] \times \mathbb{R}^{n}}|\nabla \eta|^{2}\left|\nabla_{y} \bar{u}\right|^{2} .
$$

Now, recalling (4), we have that $\nabla_{y} \bar{u} \neq 0$ in $(0,1) \times \mathbb{R}^{n}$, and so we write

$$
\begin{aligned}
& \sum_{\substack{2 \leq i \leq n+1 \\
1 \leq j \leq n+1}}\left(\partial_{X_{i} X_{j}} \bar{u}\right)^{2}-|\nabla| \nabla_{y} \bar{u}||^{2} \\
= & \sum_{\substack{2 \leq i \leq n+1 \\
1 \leq j \leq n+1}}\left(\partial_{X_{i} X_{j}} \bar{u}\right)^{2}-\left(\partial_{x}\left|\nabla_{y} \bar{u}\right|\right)^{2}-\left.\left|\nabla_{y}\right| \nabla_{y} \bar{u}\right|^{2} \\
= & \sum_{\substack{2 \leq i \leq n+1 \\
2 \leq j \leq n+1}}\left(\partial_{X_{i} X_{j}} \bar{u}\right)^{2}+\sum_{2 \leq i \leq n+1}\left(\partial_{x X_{i}} \bar{u}\right)^{2}-\left(\nabla_{y} \bar{u}_{x} \cdot \frac{\nabla_{y} \bar{u}}{\left|\nabla_{y} \bar{u}\right|}\right)^{2}-\left.\left|\nabla_{y}\right| \nabla_{y} \bar{u}\right|^{2} .
\end{aligned}
$$

Thus, we define

$$
\mathscr{Z}=\sum_{2 \leq i \leq n+1}\left(\partial_{x X_{i}} \bar{u}\right)^{2}-\left(\nabla_{y} \bar{u}_{x} \cdot \frac{\nabla_{y} \bar{u}}{\mid \nabla_{y} \bar{u}}\right)^{2} .
$$

Using the Cauchy-Schwarz inequality,

so

$$
\left(\nabla_{y} \bar{u}_{x} \cdot \frac{\nabla_{y} \bar{u}}{\left|\nabla_{y} \bar{u}\right|}\right)^{2} \leq\left|\nabla_{y} \bar{u}_{x}\right|^{2}=\sum_{2 \leq i \leq n+1}\left(\partial_{x X_{i}} \bar{u}\right)^{2}
$$

$$
\mathscr{Z} \geq 0
$$

and

$$
\mathscr{Z}=0 \text { if and only if } \nabla_{y} \bar{u}_{x} \text { is parallel to } \nabla_{y} \bar{u} \text {. }
$$

From (20), (21) and (22),

$$
\int_{[0,1] \times \mathbb{R}^{n}} \eta^{2}\left(|\mathscr{Z}|+\sum_{\substack{2 \leq i \leq n+1 \\ 2 \leq j \leq n+1}}\left(\partial_{X_{i} X_{j}} \bar{u}\right)^{2}-\left|\nabla_{y}\right| \nabla_{y} \bar{u}||^{2}\right) \leq \int_{[0,1] \times \mathbb{R}^{n}}|\nabla \eta|^{2}\left|\nabla_{y} \bar{u}\right|^{2}
$$

We now introduce some geometric notation on the level set of $\bar{u}$.

Fixed any $x_{o} \in(0,1)$ and any $c \in \mathbb{R}$, we consider the level set of $\bar{u}$ on the slice $\left\{x=x_{o}\right\}$, that is

$$
L=\left\{y \in \mathbb{R}^{n} \text { s.t. } \bar{u}\left(x_{o}, y\right)=c\right\} .
$$

Due to (4), we have that $L$ is, locally, a smooth $(n-1)$-dimensional manifold, thus we may consider its principal curvatures $\kappa_{1}, \ldots, \kappa_{n-1}$.

We define

$$
\mathscr{K}=\sqrt{\kappa_{1}^{2}+\cdots+\kappa_{n-1}^{2}} .
$$


Also, we may consider the tangential gradient $\bar{\nabla}$ along $L$. Namely, given a smooth function $G: \mathbb{R}^{n} \rightarrow \mathbb{R}$, we set

$$
\bar{\nabla} G(y)=\nabla_{y} G(y)-\left(\nabla_{y} G(y) \cdot \frac{\nabla_{y} \bar{u}\left(x_{o}, y\right)}{\left|\nabla_{y} \bar{u}\left(x_{o}, y\right)\right|}\right) \frac{\nabla_{y} \bar{u}\left(x_{o}, y\right)}{\left|\nabla_{y} u\left(x_{o}, y\right)\right|} .
$$

From Lemma 2.1 of [33], applied on the slice $\left\{x=x_{o}\right\}$, one has that

$$
\sum_{\substack{2 \leq i \leq n+1 \\ 2 \leq j \leq n+1}}\left(\partial_{X_{i} X_{j}} \bar{u}\right)^{2}-\left|\nabla_{y}\right| \nabla_{y} \bar{u}||^{2}=\left|\nabla_{y} \bar{u}\right|^{2} \mathscr{K}^{2}+|\bar{\nabla}| \nabla_{y} \bar{u}||^{2} .
$$

As a consequence, (24) becomes

$$
\int_{[0,1] \times \mathbb{R}^{n}} \eta^{2}\left(|\mathscr{Z}|+\left|\nabla_{y} \bar{u}\right|^{2} \mathscr{K}^{2}+|\bar{\nabla}| \nabla_{y} \bar{u}||^{2}\right) \leq \int_{[0,1] \times \mathbb{R}^{n}}|\nabla \eta|^{2}\left|\nabla_{y} \bar{u}\right|^{2}
$$

This geometric estimate may be seen as the extension of the weighted Poincaré inequality of $[33,34]$ that fits our goals.

Since (25) is valid for any $\eta \in C_{0}^{\infty}\left(\mathbb{R}^{n+1}\right)$, by approximation, we have that it is valid for any $\eta \in W_{0}^{1, \infty}\left(\mathbb{R}^{n+1}\right)$.

In particular, fixed $R \geq 1$, to be taken large in the sequel, we take

$$
\vartheta \in C_{0}^{\infty}\left(B_{2 R^{2}},[0,1]\right),
$$

with $\vartheta=1$ in $B_{R^{2}}$, and $\eta(x, y)=\vartheta(x, y) \tilde{\eta}(y)$, with

$$
\tilde{\eta}(y)=\left\{\begin{array}{cc}
\log R & \text { if }|y| \leq \sqrt{R}, \\
2 \log (R /|y|) & \text { if } \sqrt{R}<|y|<R, \\
0 & \text { if }|y| \geq R
\end{array}\right.
$$

We observe that, in $(0,1) \times \mathbb{R}^{n}$,

$$
|\nabla \eta(x, y)| \leq \frac{2 \chi_{[\sqrt{R}, R]}(|y|)}{|y|}
$$

as long as $R$ is large enough.

Hence, (25) yields that

$$
(\log R)^{2} \int_{[0,1] \times B_{\sqrt{R}}}\left(|\mathscr{Z}|+\left|\nabla_{y} \bar{u}\right|^{2} \mathscr{K}^{2}+|\bar{\nabla}| \nabla_{y} \bar{u}||^{2}\right) \leq \int_{[0,1] \times\{|y| \in[\sqrt{R}, R]\}} \frac{\left|\nabla_{y} \bar{u}\right|^{2}}{|y|^{2}}
$$

for large $R$.

Now, we define, for any $y \in \mathbb{R}^{n}$,

$$
g_{\star}(y)=\int_{[0,1]}\left|\nabla_{y} \bar{u}(x, y)\right|^{2} d x
$$

and, for any $\tau \geq 0$,

$$
\eta_{\star}(\tau)=\int_{|y| \leq \tau} g_{\star}(y) d y .
$$

By (5), we know that $\eta_{\star}(\tau) \leq C \tau^{2}$ as long as $\tau \geq C$. 
As a consequence, employing Lemma 3.1 of [21],

$$
\begin{gathered}
\frac{1}{2} \int_{x \in[0,1]} \int_{\sqrt{R} \leq|y| \leq R} \frac{\left|\nabla_{y} \bar{u}\right|^{2}}{|y|^{2}} d y d x=\frac{1}{2} \int_{\sqrt{R} \leq|y| \leq R} \frac{g_{\star}(y)}{|y|^{2}} d y \\
\leq \int_{\sqrt{R}}^{R} \frac{\eta_{\star}(\tau)}{\tau^{3}} d \tau+\frac{\eta_{\star}(R)}{R^{2}} \leq C(\log R+1)
\end{gathered}
$$

provided that $R \geq C$.

Therefore, (26) gives that

$$
\lim _{R \rightarrow+\infty} \int_{[0,1] \times B_{\sqrt{R}}}\left(|\mathscr{Z}|+\left|\nabla_{y} \bar{u}\right|^{2} \mathscr{K}^{2}+|\bar{\nabla}| \nabla_{y} \bar{u}||^{2}\right) \leq \lim _{R \rightarrow+\infty} \frac{16 C}{\log R}=0 .
$$

Thus,

$$
\mathscr{K} \text { vanishes identically }
$$

and so does $\mathscr{Z}$.

From (27), we have that all the principal curvatures of any sliced level set $L$ vanish.

So, there exist $U:(0,1) \times \mathbb{R} \rightarrow \mathbb{R}$ and $\omega:(0,1) \rightarrow \mathrm{S}^{n-1}$ such that

$$
\bar{u}(x, y)=U(x, \omega(x) \cdot y)
$$

for any $x \in(0,1)$ and $y \in \mathbb{R}^{n}$.

Moreover, $\nabla_{y} \bar{u}_{x}$ is parallel to $\nabla_{y} \bar{u}$, thanks to (28) and (23). This, (4) and Lemma A.1 of [5] imply that $\omega$ is constant.

Therefore

$$
u(y)=\lim _{x \rightarrow 0^{+}} \bar{u}(x, y)=\lim _{x \rightarrow 0^{+}} U(x, \omega \cdot y),
$$

which completes the proof of Theorem 1.

With this, we are now ready for the

Proof of Corollary 2. Let $\bar{u}$ be as in (1), and $u(y)=\bar{u}(0, y)$. Since $u$ is bounded, elliptic regularity theory $[23]$ gives that $|\nabla \bar{u}| \in L^{\infty}\left([0,1] \times \mathbb{R}^{2}\right)$ and so (5) holds true since $n=2$ in this case. Then, Corollary 2 plainly follows from Theorem 1 .

\section{Acknowledgments}

The work of RdlL is supported by NSF grants.

The work of EV is supported by MIUR "Variational Methods and Nonlinear Differential Equations" and FIRB "Analysis and Beyond".

This paper was written while the authors were visiting the Centre de Recerca Matemática in Bellaterra, whose warm hospitality was particularly pleasant. 


\section{References}

[1] G. Alberti, L. Ambrosio, and X. Cabré, On a long-standing conjecture of E. De Giorgi: symmetry in $3 D$ for general nonlinearities and a local minimality property, Acta Appl. Math. 65 (2001), no. 1-3, 9-33. Special issue dedicated to Antonio Avantaggiati on the occasion of his 70th birthday.

[2] X. Cabré and J. Solà-Morales, Layer solutions in a half-space for boundary reactions, Comm. Pure Appl. Math. 58 (2005), no. 12, 1678-1732.

[3] L. Caffarelli, J.-M. Roquejoffre, and Y. Sire, Free boundaries with fractional Laplacians, In preparation (2007)

[4] L. A. Caffarelli, Further regularity for the Signorini problem, Comm. Partial Differential Equations 4 (1979), no. 9, 1067-1075.

[5] M. Chermisi and E. Valdinoci, Fibered nonlinearities for $p(x)$-Laplace equations, Adv. Calc. Var. 2 (2009), no. 2, 185-205.

[6] D. Colton and R. Kress, Inverse acoustic and electromagnetic scattering theory, Vol. 93 of Applied Mathematical Sciences, Springer-Verlag, Berlin, second edition (1998), ISBN 3-54062838-X.

[7] D. Cordoba, Nonexistence of simple hyperbolic blow-up for the quasi-geostrophic equation, Ann. of Math. (2) 148 (1998), no. 3, 1135-1152.

[8] W. Craig, Non-existence of solitary water waves in three dimensions, R. Soc. Lond. Philos. Trans. Ser. A Math. Phys. Eng. Sci. 360 (2002), no. 1799, 2127-2135. Recent developments in the mathematical theory of water waves (Oberwolfach, 2001).

[9] W. Craig and M. D. Groves, Hamiltonian long-wave approximations to the water-wave problem, Wave Motion 19 (1994), no. 4, 367-389.

[10] W. Craig and D. P. Nicholls, Travelling two and three dimensional capillary gravity water waves, SIAM J. Math. Anal. 32 (2000), no. 2, 323-359 (electronic).

[11] W. Craig, U. Schanz, and C. Sulem, The modulational regime of three-dimensional water waves and the Davey-Stewartson system, Ann. Inst. H. Poincaré Anal. Non Linéaire 14 (1997), no. 5, $615-667$.

[12] W. Craig, C. Sulem, and P.-L. Sulem, Nonlinear modulation of gravity waves: a rigorous approach, Nonlinearity 5 (1992), no. 2, 497-522.

[13] W. Craig and P. A. Worfolk, An integrable normal form for water waves in infinite depth, Phys. D 84 (1995), no. 3-4, 513-531.

[14] E. De Giorgi, Convergence problems for functionals and operators, in Proceedings of the International Meeting on Recent Methods in Nonlinear Analysis (Rome, 1978), 131-188, Pitagora, Bologna (1979).

[15] R. de la Llave and P. Panayotaros, Gravity waves on the surface of the sphere, J. Nonlinear Sci. 6 (1996), no. 2, 147-167.

[16] J. J. Duistermaat and V. W. Guillemin, The spectrum of positive elliptic operators and periodic bicharacteristics, Invent. Math. 29 (1975), no. 1, 39-79.

[17] G. Duvaut and J.-L. Lions, Inequalities in mechanics and physics, Springer-Verlag, Berlin (1976), ISBN 3-540-07327-2. Translated from the French by C. W. John, Grundlehren der Mathematischen Wissenschaften, 219.

[18] A. Farina, Propriétés qualitatives de solutions d'équations et systèmes d'équations non-linéaires (2002) Habilitation à diriger des recherches, Paris VI.

[19] A. Farina, B. Sciunzi, and E. Valdinoci, Bernstein and De Giorgi type problems: new results via a geometric approach, Ann. Scuola Norm. Sup. Pisa Cl. Sci. (5) 7 (2008), no. 4, 741-791.

[20] C. Fefferman and R. de la Llave, Relativistic stability of matter. I, Rev. Mat. Iberoamericana 2 (1986), no. 1-2, 119-213.

[21] F. Ferrari and E. Valdinoci, A geometric inequality in the Heisenberg group and its applications to stable solutions of semilinear problems, Math. Ann. 343 (2009), no. 2, 351-370.

[22] G. K. Gächter and M. J. Grote, Dirichlet-to-Neumann map for three-dimensional elastic waves, Wave Motion 37 (2003), no. 3, 293-311.

[23] D. Gilbarg and N. S. Trudinger, Elliptic partial differential equations of second order, Classics in Mathematics, Springer-Verlag, Berlin (2001), ISBN 3-540-41160-7. Reprint of the 1998 edition. 
[24] M. J. Grote and C. Kirsch, Dirichlet-to-Neumann boundary conditions for multiple scattering problems, J. Comput. Phys. 201 (2004), no. 2, 630-650.

[25] B. Hu and D. P. Nicholls, Analyticity of Dirichlet-Neumann operators on Hölder and Lipschitz domains, SIAM J. Math. Anal. 37 (2005), no. 1, 302-320 (electronic).

[26] N. S. Landkof, Foundations of modern potential theory, Springer-Verlag, New York (1972). Translated from the Russian by A. P. Doohovskoy, Die Grundlehren der mathematischen Wissenschaften, Band 180.

[27] A. J. Majda and E. G. Tabak, A two-dimensional model for quasigeostrophic flow: comparison with the two-dimensional Euler flow, Phys. D 98 (1996), no. 2-4, 515-522. Nonlinear phenomena in ocean dynamics (Los Alamos, NM, 1995).

[28] P. I. Naumkin and I. A. Shishmarëv, Nonlinear nonlocal equations in the theory of waves, American Mathematical Society, Providence, RI (1994), ISBN 0-8218-4573-X. Translated from the Russian manuscript by Boris Gommerstadt.

[29] D. P. Nicholls and M. Taber, Joint analyticity and analytic continuation of Dirichlet-Neumann operators on doubly perturbed domains, J. Math. Fluid Mech. 10 (2008), no. 2, 238-271.

[30] O. Savin and E. Valdinoci, Elliptic PDEs with fibered nonlinearities, J. Geom. Anal. 19 (2009), no. 2, 420-432.

[31] Y. Sire and E. Valdinoci, Fractional Laplacian phase transitions and boundary reactions: a geometric inequality and a symmetry result, J. Funct. Anal. 256 (2009), no. 6, 1842-1864.

[32] E. M. Stein, Singular integrals and differentiability properties of functions, Princeton University Press, Princeton, N.J. (1970). Princeton Mathematical Series, No. 30.

[33] P. Sternberg and K. Zumbrun, Connectivity of phase boundaries in strictly convex domains, Arch. Rational Mech. Anal. 141 (1998), no. 4, 375-400.

[34] — A Poincaré inequality with applications to volume-constrained area-minimizing surfaces, J. Reine Angew. Math. 503 (1998) 63-85.

[35] J. J. Stoker, Water waves: The mathematical theory with applications, Pure and Applied Mathematics, Vol. IV, Interscience Publishers, Inc., New York (1957).

[36] E. Valdinoci, Flatness of Bernoulli jets, Math. Z. 254 (2006), no. 2, 257-298.

[37] G. B. Whitham, Linear and nonlinear waves, Wiley-Interscience [John Wiley \& Sons], New York (1974). Pure and Applied Mathematics.

[38] V. Zakharov, Stability of periodic waves of finite amplitude on the surface of a deep fluid, Zh. Prikl. Mekh. Tekh. Fiz. 9 (1968) 86-94.

University of Texas at Austin, Department of Mathematics, 1 University Station C1200, Austin, TX 78712-0257 (USA)

E-mail address: llave@math.utexas.edu

Dipartimento di Matematica, Università di Roma Tor Vergata, Via della Ricerca SciENTIFICA, 1, I-00133 Roma (Italy)

E-mail address: enrico@math.utexas.edu 